\title{
The effect of Helium-enhanced stellar populations on the ultraviolet upturn phenomenon of early-type galaxies
}

\section{Chul Chung, Suk-Jin Yoon and Young-Wook Lee}

Center for Galaxy Evolution Reasearch and Department of Astronomy,

Yonsei University, Seoul 120-749, Republic of Korea

email: chung@galaxy.yonsei.ac.kr

\begin{abstract}
We present new population synthesis models (Chung et al. 2011) for quiescent earlytype galaxies (ETGs) with UV-upturn phenomenon using relatively metal-poor and heliumenhanced subpopulations in the model. We find that the presence of helium-enhanced subpopulations in ETGs can naturally reproduce the strong UV-upturns observed in giant elliptical galaxies (Figure 1. left panel), without invoking unrealistically old ages (Park \& Lee 1997). Our models with helium-enhanced subpopulations also predict that the well-known Burstein relation can be explained by the fraction of helium-enhanced subpopulation, the mean age, and the mean metallicity of the underlying stellar populations (Figure 1. right panel).
\end{abstract}

Keywords. galaxies: elliptical and lenticular, CD - galaxies: evolution - galaxies: stellar content - ultraviolet: galaxies
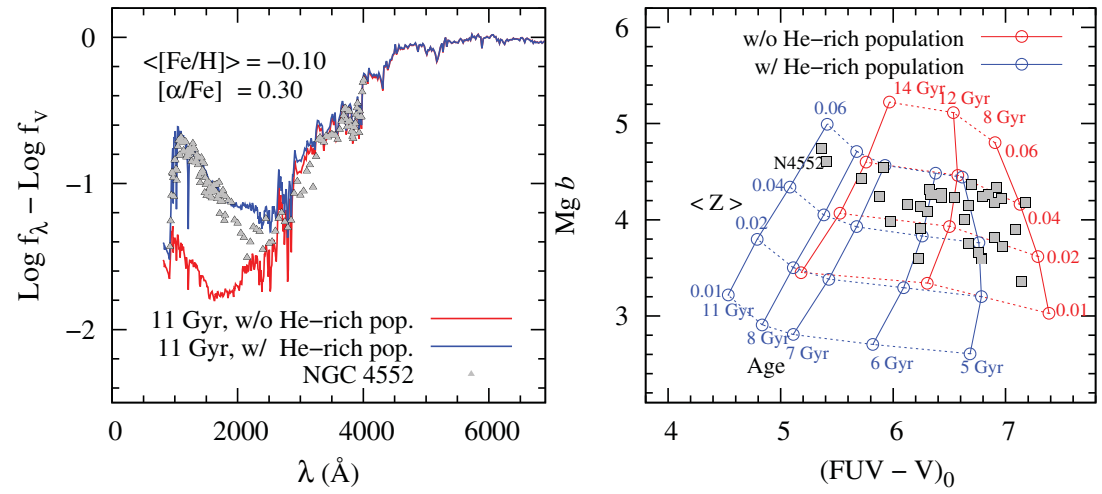

Figure 1. (Left) Comparison of observed SEDs of NGC 4552 with our models. (Right) FUV-V color vs. Mg b correlation for the sample of quiescent ETGs from Bureau et al. (2011).

\section{References}

Bureau, M., et al. 2011, MNRAS, 414, 1887

Chung, C., Yoon, S.-J., \& Lee, Y.-W. 2011, ApJ (Letters) 740, L45

Park, J.-H. \& Lee, Y.-W. 1997, ApJ, 476, 28 